\title{
Repeat-station surveys: implications from chaos and ergodicity of the recent geomagnetic field
}

\author{
Angelo De Santis ${ }^{1,2,}$, Enkelejda Qamili ${ }^{1,3}$, Gianfranco Cianchini ${ }^{1,3}$ \\ ${ }^{1}$ Istituto Nazionale di Geofisica e Vulcanologia, Sezione Roma 2, Roma, Italy \\ ${ }^{2}$ Università "G. D'Annunzio" di Chieti-Pescara, Campus Universitario, Chieti, Italy \\ ${ }^{3}$ Università di Siena, Scuola di Dottorato in Scienze Polari, Siena, Italy
}

\section{Article history}

Received November 30, 2011; accepted April 2, 2012.

\section{Subject classification:}

Instruments and techniques, Global and regional models, Main geomagnetic field, Mathematical geophysics, Statistical analysis.

\begin{abstract}
The present geomagnetic field is chaotic and ergodic: chaotic because it can no longer be predicted beyond around 6 years; and ergodic in the sense that time averages correspond to phase-space averages. These properties have already been deduced from complex analyses of observatory time series in a reconstructed phase space [Barraclough and De Santis 1997] and from global predicted and definitive models of differences in the time domain [De Santis et al. 2011]. These results imply that there is a strong necessity to make repeat-station magnetic surveys more frequently than every 5 years. This, in turn, will also improve the geomagnetic field secular variation models. This report provides practical examples and case studies.
\end{abstract}

\section{Introduction and motivation}

Repeat-station surveys [e.g., Barraclough and De Santis 2011] are important, among other things for: (a) modeling the main geomagnetic field and global and regional secular variation; (b) navigational purposes; and (c) better definition of magnetic anomalies. Two issues are fundamental for the present report: the first regards the repeat time of site occupation in the repeat-station surveys; and the second concerns the kind of data analysis we perform at the repeat station.

Generally, the typical time interval of re-occupation of a repeat station is around 5 years. However, there has been recent debate in the geomagnetism community about whether this time lag should be reduced or maintained. In addition, the measurement values considered during the repeat-station reoccupation are temporal averages of all the measurements at the same site. These values are then correctly reduced, taking into account a close observatory or a fixed reference station [e.g., Barraclough and De Santis 2011]. Alternative schemes of analysis and averages have also been proposed. However, we can ask whether these simple averages are still appropriate or not. The aim of this report is to justify on the basis of the physics of the recent geomagnetic field: (i) more frequent repeat-station survey re-occupation; and (ii) the present typical operation of time averaging at each repeat station.

To do this, we will first introduce some concepts on chaos and ergodicity, and then we will recall some previous results from analyses of recent geomagnetic field data and models, which provide evidence for both of these physical properties of the field. When we transfer these results to magnetic repeat-station surveys, we arrive at the clear conclusion that more frequent occupations are a real necessity according to a solid physical basis, instead of the current interval of 5 years. Finally, we complete the report with some conclusions.

\section{Chaos theory}

Chaos theory studies dynamical systems where the behavior is highly sensitive to the initial conditions. If we consider the time evolution $x(t)$ of a nonlinear process, it is possible to represent this by Equation (1) for continuous time $t$ :

$$
\dot{\vec{x}}(t)=\overrightarrow{\mathrm{F}}(\vec{x}(t)), t \in
$$

or by Equation (2) for discrete time:

$$
x_{n+1}=f\left(x_{n}\right), n \in
$$

where, $F$ and $f$ are some nonlinear functions of $x$.

As in Barraclough and De Santis [1997] and De Santis et al. [2002], we make use of the concept of phase space to characterize the dynamic system under study. This phasespace diagram is basically an abstract reference system, the coordinates of which are all the independent variables that are necessary to completely describe the state of the system at any moment with just a point. For instance, let us consider the solution of the discrete case; i.e., the solution of Equation (2), with the initial state $x_{0}$ : the sequence $x_{n}=f^{n}\left(x_{0}\right)$ is a trajectory of the phase-space diagram; i.e., the evolu- 
tion with time of the system under dynamics $f$. When a real system is under consideration, the problem is the absence of information about all of the variables involved. In this case, a way to represent the system dynamics is through an appropriate phase-space reconstruction. This operation, which is a local approximation approach, allows the reconstruction (or embedding) of a single-dimensional time series into a multi-dimensional phase space. In this way, the system dynamics is represented locally step-by-step in this new phase space.

A system is chaotic if each small change $\varepsilon$ of some initial close trajectory in the phase space propagates exponentially with time $t$; i.e.:

$$
\mathcal{E}(t) \approx \varepsilon_{0} \cdot \exp (K \cdot t)
$$

This is just the exponential behavior in time of $\varepsilon(t)$ that will lead us to investigate in greater detail in section 5 the possible implications in repeat-station surveys. The Kolmogorov entropy, $K(>0)$, measures the rate of information

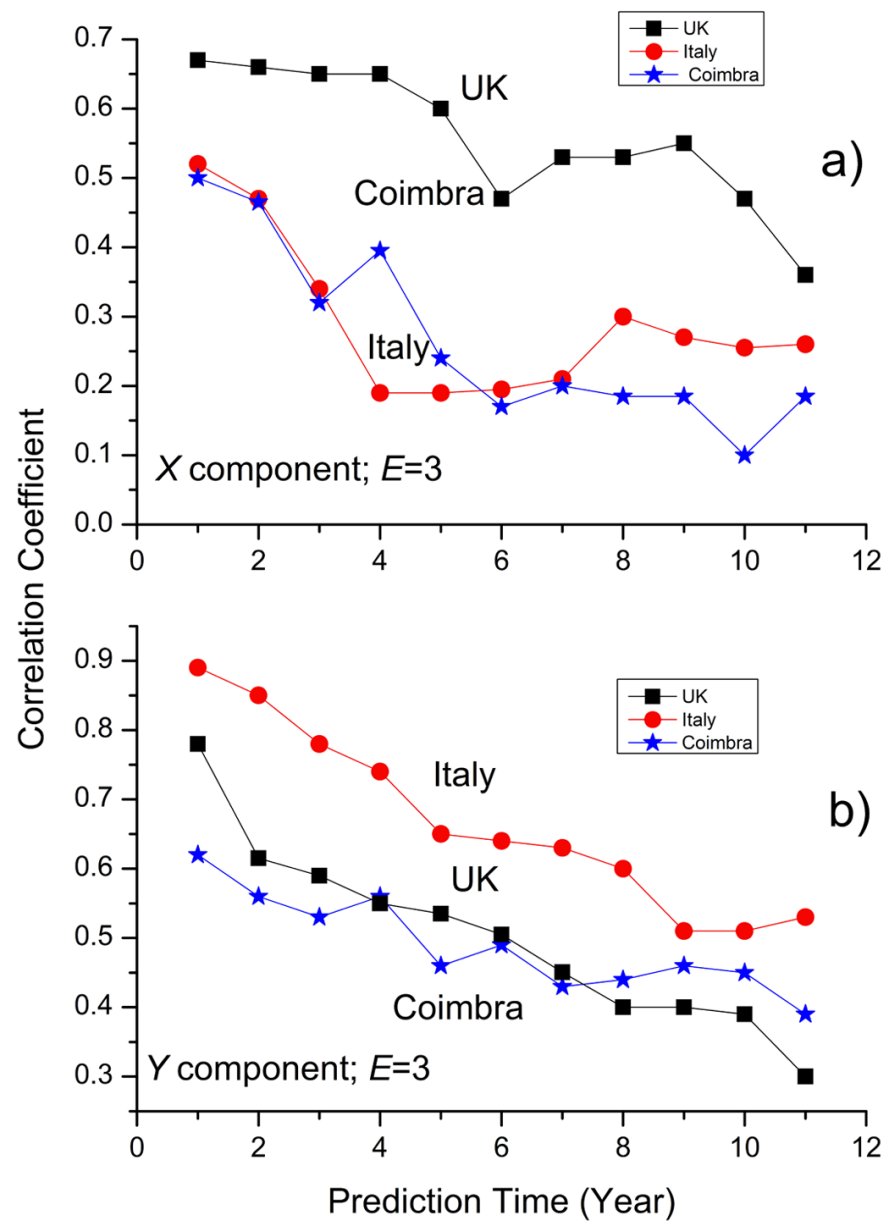

Figure 1. Correlation coefficients between the predicted (a) and observed (b) values of the $X$ and $Y$ geomagnetic time series of three European observatories when reconstructed in a phase space (adapted from Barraclough and De Santis [1997]). The decrease in time is a symptom for a chaotic geomagnetic field. $E=3$ is the embedding dimension of the reconstructed phase space. loss of the system under study [Schuster and Just 2005], and it is inversely proportional to the time over which a chaotic system is predictable. For this reason, we can introduce here the mean time $<T>=1 / K$ after which no reliable prediction can be made [Barraclough and De Santis 1997, De Santis et al. 2002]. As described above, a way to represent such a dynamic system is through phase-space reconstruction. One of the most popular methods available for reconstructing the phase space from an experimental time series is that of time delays that was introduced by Takens [1981]. By using the past history and an appropriate delay time, $\tau$, this method can allow us to project a time series $x_{i}$ into a reconstructed $E$-dimensional phase space $y_{i}$, such that:

$$
y_{i}=\left[x\left(t_{i}\right), x\left(t_{i}+\tau\right), \ldots, x\left[t_{i}+(E-1) \tau\right]\right.
$$

where, $i=1,2, \ldots, N, N$ is the number of data points of the state variable $x(t), \mathrm{E}$ is the so-called embedding dimension, which is greater than or equal to the number of degrees of freedom of the dynamic system, and also to the number of deterministic scalar equations that characterize it.

Once this pseudo-space is reconstructed, it is simple to check whether there is divergence of a perturbed trajectory with respect to the original one. Almost all of the techniques used to detect chaos in time series require a large amount of data. A simple way that overcomes this limitation is to apply a nonlinear forecasting approach in the phase space [Wales 1991, Sugihara and May 1990]. With this method, we split the sequence under study into two parts, and use the first part to predict the second one: the comparison between the predicted and observed values allows us to deduce some important properties about the possible chaoticity of the time series. When applied to several geomagnetic observatory time series, Barraclough and De Santis [1997] and De Santis et al. [2002] found a clear chaotic nature of the geomagnetic field with $\langle T\rangle=6$ years (Figure 1). We will show below how this result, together with the ergodic property of the field, is important for the frequency of repeat-station re-occupation.

\section{Ergodicity}

Historically, the issue of ergodicity arose from the work of Boltzmann on the determination of average values in kinetic theory. For a given dynamic system represented by $(\Omega$, $f^{t}, r$ ) in the phase space $\Omega$ with an invariant (during evolution) measure $r$, a deterministic dynamics $f^{t}$ drives the time evolution along a path (named the trajectory) that will pass through all of the possible states the system can reach, for a sufficiently long time. So, this behavior should allow the substitution of the phase-space average with the temporal one of the same observable. This is the ergodic hypothesis [Eckmann and Ruelle 1985].

As a formula, it translates into [e.g., Walters 1982]: 


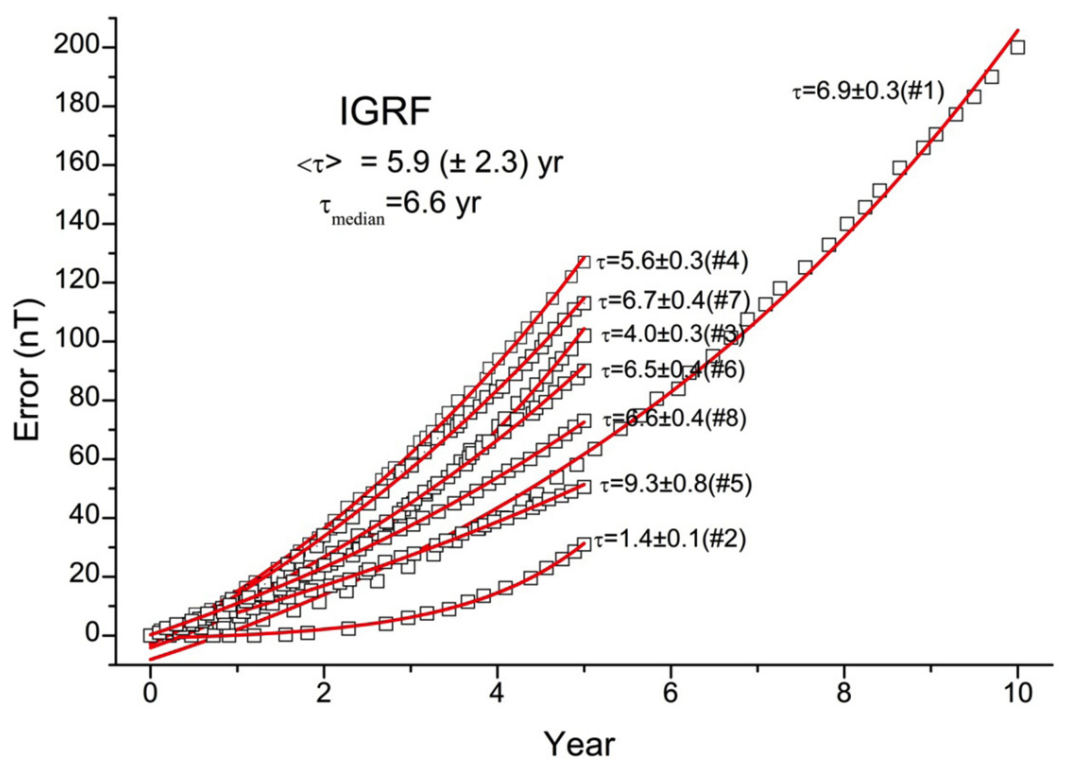

Figure 2. Error behavior between the predicted and observed global International Geomagnetic Reference Field (IGRF) models (from De Santis et al. [2011]). The exponential increase in time is a symptom of a chaotic geomagnetic field.

$$
\left\langle A\left(x_{0}\right)\right\rangle=\lim _{t \rightarrow \infty} \frac{1}{T} \int_{0}^{T} \varphi\left[f^{t}\left(x_{0}\right)\right] d t=\int \rho(d t) \varphi(x)=\underline{A}
$$

As a consequence of this feature, if the system is ergodic and $A(x)$ is an observable, the time average $<A\left(x_{0}\right)>$ does not depend on the initial state $x_{0}$; i.e., after a certain time evolution, the system is no longer dependent on its initial state. $\underline{A}$ is an appropriate average in the phase space.

\section{Chaotic and ergodic character of the recent geomag- netic field}

As mentioned above, some previous studies have confirmed the chaotic properties of the recent geomagnetic field [Barraclough and De Santis 1997, De Santis et al. 2002, De Santis and Qamili 2010], i.e., its sensitivity to initial conditions in the phase space.

When the process under study is ergodic, the phasespace reconstruction is not necessary, and we can perform the analyses in the time domain. De Santis et al. [2011] demonstrated that this is the case of the geomagnetic field. Under the ergodic condition, a good technique used to find possible nonlinearity and chaos in a system is a simple nonlinear forecasting approach in the time domain. This technique uses the past history of a time series to forecast future values, without reconstructing the phase space. This result was determined by De Santis et al. [2011] through an analysis of the divergence of the errors between the predicted and definitive global geomagnetic models, with the estimation of the typical mean time $\langle\tau\rangle$ after which time no prediction is reliable. They found $\langle\tau\rangle=6$ years, which is the same time average $<T>$ that results from the analysis with phasespace reconstruction (Figure 2). This confirms that it is not possible to predict the evolution into the future of the geomagnetic secular variation by more than 6 years, so that any prediction must be limited to a shorter time, whereby the shorter this time, the better the prediction will be.

We argued in De Santis et al. [2011] that the close agreement between the outcomes from two independent analyses, i.e., $\langle\tau\rangle$ as deduced from the time-error analysis in the time domain, and $\langle T\rangle$ as estimated from the nonlinear forecasting approach in the phase space [De Santis et al. 2002], confirms the chaotic nature of the geomagnetic field and represents empirical proof of its ergodicity.

It could be said that the concepts of chaos and ergodicity seem in contradiction, but this is only apparently so: there are many examples of chaotic phenomena that are also ergodic [e.g., Eckman and Ruelle 1985]. This can be understood also for the geomagnetic dynamo: although in general it has nonequilibrium and nonstationary dynamics, the geodynamo can evolve into parts of the phase space that are characterized by both chaos and ergodicity [e.g., Vincent 2005, Shen et al. 2009]. There are other evident nonequilibrium and nonstationary geophysical phenomena that can show analogous properties: e.g., seismic sequences have been shown to be ergodic [Tiampo et al. 2007] and chaotic [De Santis et al. 2010] occasionally.

The properties of chaos and ergodicity of the recent geomagnetic field, i.e., the short predictability in time and the sort of space-time regularity, respectively, allow us to make reasonable forecasts of the geomagnetic field for a few years in advance; e.g., to update the geomagnetic charts for a few years into the future. Also, shorter time variations of the geomagnetic field that are due to external contributions that arise mainly from the ionosphere and magnetosphere have shown important chaotic characteristics [e.g., Balasis et al. 2010], although this aspect will not be considered in this report, which is mainly concerned with the corresponding chaotic properties of the internal geomagnetic field and its secular variation. 


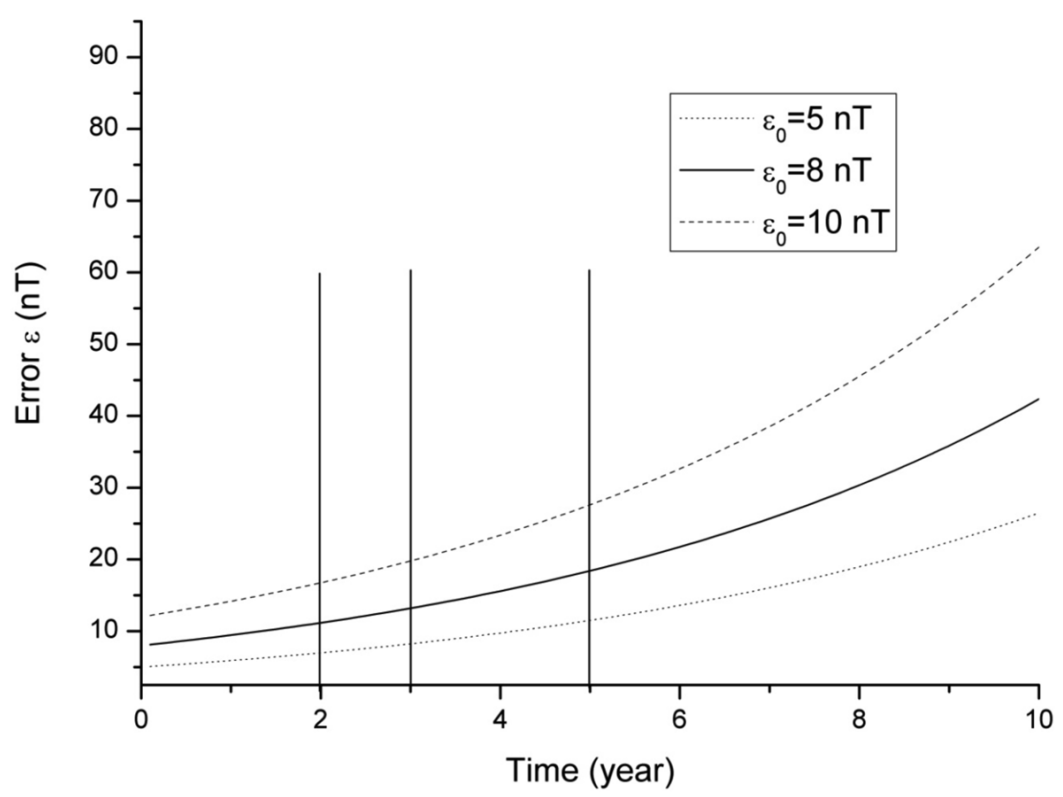

Figure 3. Exponential divergence in time of some typical initial errors (of $5 \mathrm{nT}, 8 \mathrm{nT}$ and $10 \mathrm{nT}$ ) in repeat-station surveys in the case of a chaotic geomagnetic field. Vertical lines are taken at 2 years, 3 years and 5 years. While the error after 5 years is relatively large, those at the first two epochs are accepted for any extrapolation in time of eventual magnetic charts.

\section{Consequences of repeat-station surveys}

We can now take advantage of the established properties of chaos and ergodicity of the recent geomagnetic field. In this case, Equation (3) is valid for the error $\varepsilon$ of any geomagnetic element of the field, the initial error value of which is taken as $\varepsilon_{0}$. This means that:

$$
\varepsilon(t) \approx \varepsilon_{0} \cdot \exp (K \cdot t)=\varepsilon_{0} \cdot \exp \left[\frac{t}{\langle\tau\rangle}\right]
$$

where, on the right-hand side we have replaced $K$ entropy with the reverse of the mean time of predictability $\langle\tau\rangle$. From the results given by Barraclough and De Santis [1997] and by De Santis et al. [2011], we insert $\langle\tau\rangle=\langle T\rangle=6$ years into Equation (3a) and apply it to some typical cases of repeat-station measurement errors. Equation (3a) indicates how an initial error of a certain measurement taken at the repeat station in a given epoch $t_{0}$ (here set equal to zero, for convenience) will explode after some time $t$ following the exponential law of typical chaotic processes. Figure 3 shows this temporal behavior for three possible cases of initial errors, of $5 \mathrm{nT}, 8 \mathrm{nT}$ and $10 \mathrm{nT}$; these values are reasonable values of the errors likely to be involved in the deduced values from measurements at a repeat station, including the process of time and space reduction [e.g. Meloni et al. 1994; Barraclough and De Santis 2011]. In Figure 3, vertical lines are drawn at 2, 3 and 5 years after the initial measurement: the corresponding intersections are the estimated errors at each successive epoch. It is evident that after 5 years from the initial measurement the error is relatively large, while the errors after 2 years and 3 years are still reasonably acceptable. Indeed, just to give an idea, an initial error of $8 \mathrm{nT}$ will grow to a final error of $18.5 \mathrm{nT}$ after 5 years, but will only be $11 \mathrm{nT}$ to $13 \mathrm{nT}$ after 2- years to 3 years, values which are lower (and probably still sustainable) than the error obtained after 5 years.

To better reproduce the real case of repeat-station time series at different frequencies of re-occupation, we considered also the CM4 geomagnetic model (see Sabaka et al. [2004] for details of this model) and synthesized the yearly values of each geomagnetic component $G\left(t_{i}\right)^{C M 4}\left(=X^{C M 4}\right.$, $Y^{C M 4}$ or $\left.Z^{C M 4} ; t_{i}=1960, \ldots, 2002\right)$ in the central points of Spain (Figure 4, left side) and Australia (Figure 5, left side). We chose these two ideal sites because they are eventually representative of two different regions on the Earth. For each time series, we then considered the cases of 2-year and 6-year repeat-station re-occupation. The latter was chosen (instead of simply a $1 / 5$ year $^{-1}$ frequency) for an easier one-to-one comparison with the other shorter period of re-occupation. For both frequencies of re-occupation, we calculated the following differences:

where:

$$
G\left(t_{i}\right)^{C M 4}-G\left(t_{i}\right)^{\text {pred }}
$$

$$
\begin{aligned}
G\left(t_{i}\right)^{\text {pred }} & =G\left(t_{i-1}\right)^{C M 4}+\left[G\left(t_{i-1}\right)^{C M 4}-G\left(t_{i-2}\right)^{C M 4}\right]= \\
& =2 G\left(t_{i-1}\right)^{C M 4}-G\left(t_{i-2}\right)^{C M 4}
\end{aligned}
$$

with $t_{i}$ starting at 1964 (with initial $t_{i-1}=1962$ and $t_{i-2}=$ 1960) for the 2-year re-occupation, and at 1972 for the 6-year re-occupation (with initial $t_{i-1}=1966$ and $t_{i-2}=1960$ ). Equation (7) is the extrapolation of the $G$ component at time $t_{i}$ as deduced from the previous two data points taken at times $t_{i-1}$ and $t_{i-2}$, i.e., at the epochs of the previous two magnetic surveys. Therefore, Equation (6) measures the deviation of the predicted value $G\left(t_{i}\right)^{\text {pred }}$ with respect the actual synthe- 

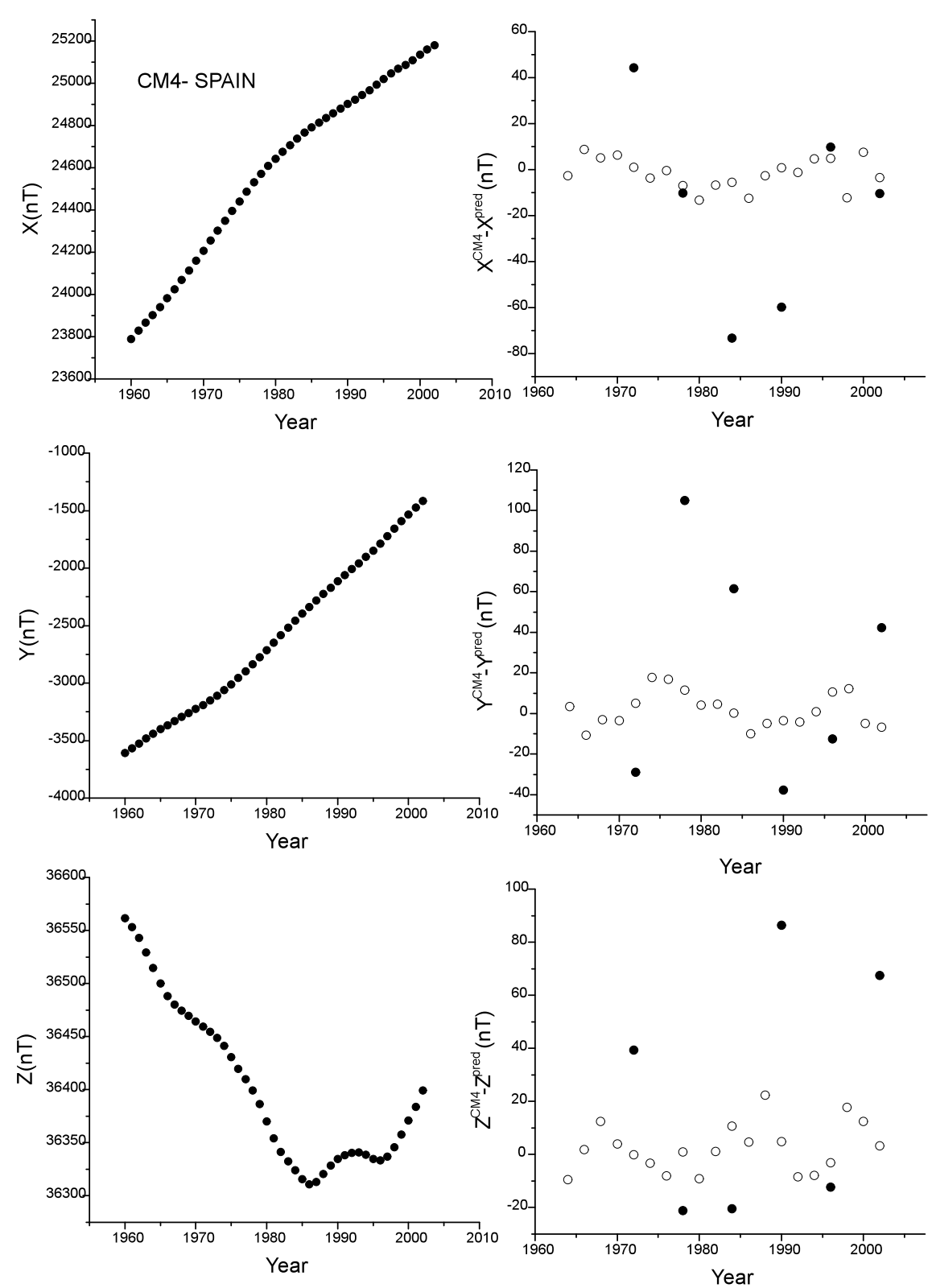

Figure 4. Left: Synthetic case of possible repeat-station time series of the geomagnetic component in a central point in Spain, as deduced from the CM4 model. Right: Differences between the synthetic (actual) values and the predicted values when the frequency of repetition is one data point every 2 years (white circles) and 6 years (black circles).

sized value from the model $G\left(t_{i}\right)^{C M 4}$. Figures 4 and 5 (right sides) show the differences expressed by Equation (6) for Spain and Australia, respectively, where the white circles represent the data points deduced with the 2-year re-occupation, and black circles are those obtained from the 6-year re-occupation. It is clear from Figures 4 and 5 that the longer time of re-occupation provides much more scattered values with expected errors that are much larger than those at the 2-year reoccupation: for instance for the $X$ component, in Spain and Australia the largest errors reach around $70 \mathrm{nT}$ and $130 \mathrm{nT}$, respectively, while they are no larger than $20 \mathrm{nT}$ for the shorter time of re-occupation. This confirms that, too long a time interval between two subsequent surveys will cause very large extrapolation errors, thus invalidating any possibility of reconstructing reliable magnetic maps in the time in between.
These simple examples show that a reduction to 2 years to 3 years for the time of re-occupation of the repeat-station network is to be recommended, to avoid the error of the temporal extrapolation from becoming too large. We believe that the present technology and potential of most European scientific institutions would allow this recommendation to be put into practice. It is also clear that any improvement in terms of error reduction in repeat-station surveys will also have positive effects on any secular variation model that is based on the repeat-station data.

However, since we understand the difficulties that some countries might have, it is not necessary for all of the repeat stations of a country to be more frequently reoccupied. A reasonable compromise could be to increase the frequency of reoccupation only in a subset (say, $10 \%$ to $20 \%$ ) of the nor- 

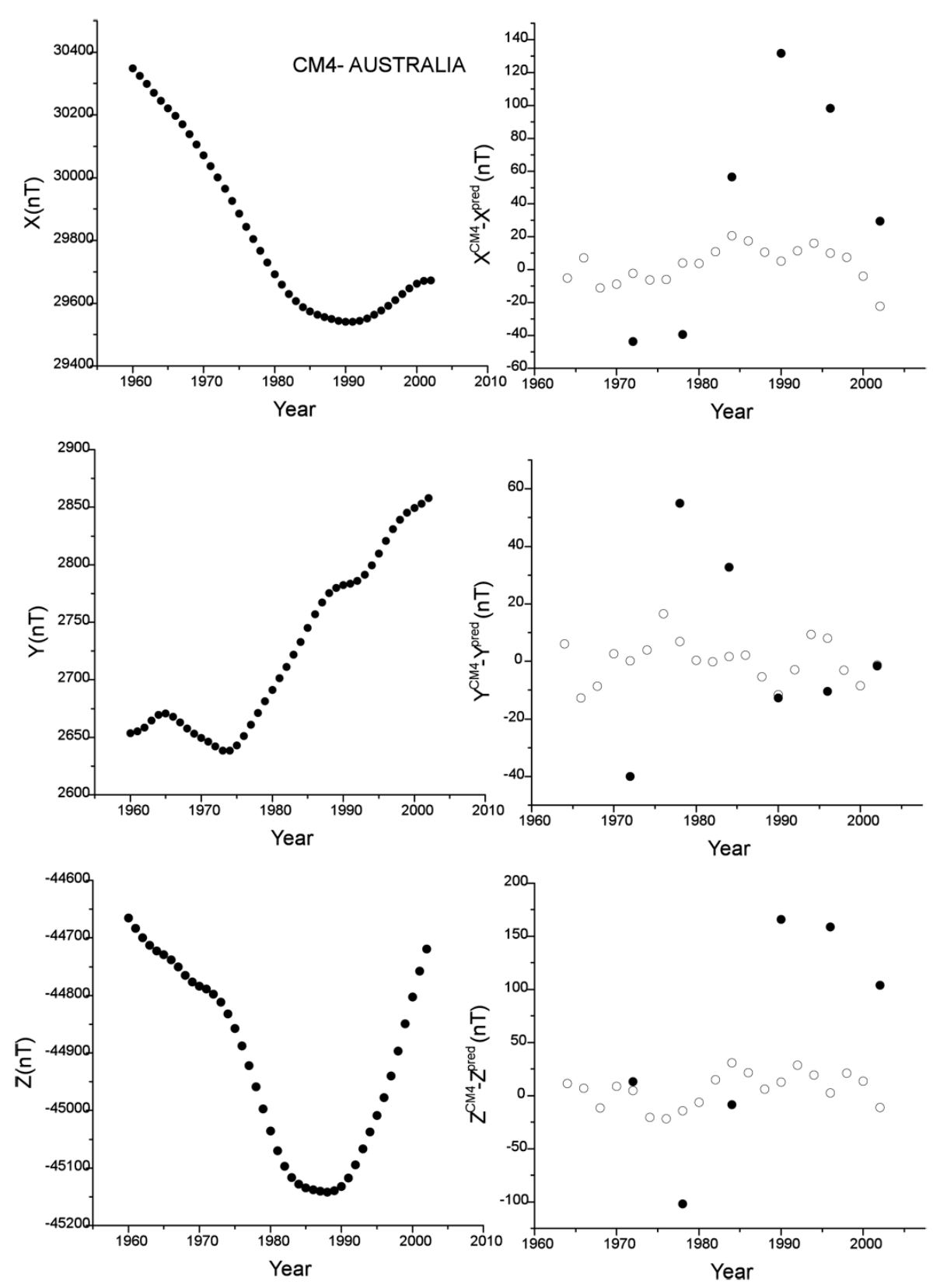

Figure 5. Left: Synthetic case of possible repeat-station time series of the geomagnetic component in a central point in Australia, as deduced from the CM4 model. Right: Differences between the synthetic (actual) values and the predicted values when the frequency of repetition is one data point every 2 years (white circles) and 6 years (black circles).

mal repeat stations (the 'super stations', as used by McEwin [1993]). In this way, it will be easier to minimize the possible errors of extrapolation between two epochs of reoccupation without too much effort.

In addition, the ergodicity of the recent geomagnetic field confirms the use of temporal arithmetic means for the estimation of the mean value of some magnetic element at a certain epoch, and the reduction in the value using corresponding temporal means at a close magnetic observatory or reference station. This is also valid for the calculation of other successive statistical moments (e.g., variance, skewness, kurtosis). The overall set of typical operations on repeat-station data made in time to reduce them to some fixed past or future epoch (i.e., averaging the daily measurements, reducing to the closest night, and then reducing to the closest observatory at, say, the middle of the year) can be performed, because we implicitly assume that the magnetic field is ergodic. Otherwise, more complex (sometime even impossible) operations would be necessary, with most of them made in the phase space, and with the implied difficulties of its reconstruction.

\section{Conclusions}

The nonlinear forecasting approach applied to the geomagnetic field in the phase space has highlighted its chaotic nature that is characterized by a mean time $\langle T\rangle=6$ years of predictability [Barraclough and De Santis 1997, De Santis et al. 2002]. At the same time, the analyses of differences be- 
tween predicted and definitive geomagnetic models in natural time and space domains provided the same result $\langle\tau\rangle=$ $6 \pm 3$ years [De Santis et al. 2011]. This supports our knowledge of the chaotic nature of the field and demonstrates its ergodicity. According to our previous results, if we want to reproduce a correct secular variation, frequent repeat-station surveys are compulsory, with, of course, the period of time of re-occupation of less than 6 years, which represents an upper limit, for the reasons we have given. Consistent with the availability of resources, a re-occupation every 2 years to 3 years is highly recommended, so that the error is less than twice the initial error. In this way, the chaotic nature of the field does not have too large an effect on the possible shorttime extrapolation of the field from the repeat-station observations. As already indicated [Newitt et al. 1996], another advantage of frequent re-occupation will be to eventually track rapid changes in secular variation, such as the jerks [e.g., Duka et al. 2012]. Finally, the property of ergodicity confirms that both the operation of making time averages at the repeat station and the comparison with analogous time averages at close magnetic observatories or stations for temporal reduction, are appropriate, because of the validity between the exchange of phase space and time averages, as given by Equation (5).

Since this report was presented at the MagnetE Workshop held in Rome, from May 9-11, 2011, we hope that our arguments have been taken into due considerations by the world-wide community of repeat-station planning and management, and in particular by the institutions contributing to the European Repeat-Station Network.

Acknowledgements. Part of the study by EQ and GC was carried out while they were attending $\mathrm{PhD}$ courses at Siena University. Some funds were supplied by a PNRA project ("Reversing Earth Magnetism") and a PRIN 2008 MIUR-funded project ("Animal Magnetic Homing", SIMMAG Unit of Research). This report was originally presented at the MagnetE Workshop held in Rome, from May 9-11, 2011. We thank the local organizers, the scientific committee, and all of the participants at the Workshop for their useful comments and suggestions that have helped us to improve the quality of this study.

\section{References}

Balasis G., I.A. Daglis, A. Anastasiadis and K. Eftaxias (2010). Investigating magnetospheric dynamics using various complexity measures, AIP Conf. Proc., 1320, 65-71.

Barraclough, D.R., and A. De Santis (1997). Some possible evidence for a chaotic geomagnetic field from observational data, Phys. Earth Plan. Inter., 99, 207-220.

Barraclough, D.R., and A. De Santis (2011). Repeat Stations Activities, In: M. Korte and M. Mandea (eds.), Geomagnetic Observations and Models, Springer, 45-55.

De Santis, A., D.R. Barraclough and R. Tozzi (2002). Nonlinear variability in the geomagnetic secular variation of the last 150 years, Fractals, 10, 297-303.

De Santis, A., and E. Qamili (2010). Shannon information of the geomagnetic field for the past 7000 years, Nonlinear Proc. Geoph., 17, 77-84.

De Santis, A., G. Cianchini, E. Qamili and A. Frepoli (2010). The 2009 L'Aquila (central Italy) seismic sequence as a chaotic process, Tectonophysics, 496, 44-52.

De Santis, A., E. Qamili and G. Cianchini (2011). Ergodicity of the recent geomagnetic field, Phys. Earth Plan. Inter, 186, 103-110.

Duka, B., A. De Santis, M. Mandea, A. Isac and E. Qamili (2012). Geomagnetic jerks characterization via spectral analysis, Solid Earth, 3, 131-148.

Eckmann, J.P., and D. Ruelle (1985). Ergodic theory of chaos and strange attractors, Rev. Mod. Phys., 57, 617-656.

McEwin, A.J. (1993). The repeat station network and estimation of secular variation in the Australian region, Explor. Geophys., 24, 87-88.

Meloni, A., O. Battelli, A. De Santis and G. Dominici (1994). The 1990.0 magnetic repeat station survey and normal reference fields for Italy, Annals of Geophysics, 37 (5), 949-967.

Newitt, L.R., C.E. Barton and J. Bitterly (1996). Guide for Magnetic Repeat Station Surveys, International Association of Geomagnetism and Aeronomy, 129 pp.

Sabaka, T.J., N. Olsen and M.E. Purucker (2004). Extending comprehensive models of the Earth's magnetic field with Ørsted and CHAMP data, Geophys. J. Int., 159, 521-547.

Schuster, H.G., and W. Just (2005). Deterministic Chaos: an Introduction (4th ed.), Wiley-VCH, Weinheim, $287 \mathrm{pp}$.

Shen, W., C. Fang and D. Zhang (2009). Fractal and chaos research of geomagnetic polarity reversal, Earth Sc. Frontiers, 16, 201-206.

Sugihara, G., and R.M. May (1990). Nonlinear forecasting as a way to distinguish chaos from measurement error in time series, Nature, 344, 734-741.

Takens, F. (1981). Detecting strange attractors in turbulence, In: D.A. Rand and L.S. Young (eds.), Lecture Notes in Mathematics, 898, Springer, Berlin, 366 pp.

Tiampo, K.F., J.B. Rundle, W. Klein, J. Holliday, J.S. Sa' Martins and C.D. Ferguson (2007). Ergodicity in natural earthquake fault networks, Phys. Rev. E, 75, 1-15.

Vincent, U.E. (2005). Synchronization of Rikitake chaotic attractor using active control, Physics Lett. A, 343, 133-138.

Wales, D.J. (1991). Calculating the rate of loss information from chaotic time series by forecasting, Nature, 350, 485-488.

Walters, P. (1982). An Introduction to Ergodic Theory, Springer, New York.

\footnotetext{
${ }^{\star}$ Corresponding author: Angelo De Santis,

Istituto Nazionale di Geofisica e Vulcanologia, Sezione Roma 2, Roma, Italy; email: angelo.desantis@ingv.it.

(C) 2013 by the Istituto Nazionale di Geofisica e Vulcanologia. All rights reserved.
} 